\title{
Exact Solution of Linear and Non-linear Goursat Problems
}

\author{
Jamshad Ahmad ${ }^{1, *}$, Mariyam Mushtaq ${ }^{2}$ \\ ${ }^{1}$ Department of Mathematics, Faculty of Sciences, University of Gujrat, Pakistan \\ ${ }^{2}$ Department of Mathematics, NCBA\&E, Gujrat (Campus), Pakistan
}

Copyright (C) 2015 Horizon Research Publishing All rights reserved.

\begin{abstract}
In this research paper, we applied the Adomian's decomposition method to determine the analytical exact solutions of linear and nonlinear Goursat problems which play very important part in applied and engineering sciences. The proposed technique is fully compatible with the complexity of these problems and obtained results are highly encouraging. Some examples with closed form solutions are studied in detail to further illustrate the proposed technique, and the results obtained indicate this approach is indeed practical and efficient.
\end{abstract}

Keywords Nonlinear Problems, Goursat Problems, Adomian's Decomposition Method

\section{Introduction}

Most of the problems in natural and engineering sciences are modeled by differential equations. These equations arise in various scientific models such as the fluid mechanics, chemical reaction diffusion, propagation, of shallow water waves, Schrodinger wave equation models. The Goursat partial differential equation arises in linear and non linear partial differential equations with mixed derivatives in the study of wave phenomena. This equation is a second order hyperbolic partial differential equation which occurs in various fields of study such as in engineering, physics, and applied mathematics. Goursat problems are known to arise in a variety of physical phenomenon and applied sciences [1-6]. Several techniques including Runge-Kutta, decomposition, finite difference, finite element, geometric mean averaging of the functional values and variational iteration have been used to investigate these problems [1-6]. Adomian's Decomposition Method was introduced by Adomian's in $[7,8]$ and used heavily in the literature to solve the wide class of physics and engineering problems such as non-linear differential equation, non-linear dynamic system, coupled non-Linear differential equations, linear and non-linear integro-differential equation and Airy's equation successfully [9-16]. This method does not require the presence of small parameters in the differential equation, and provides the solution (or an approximation to it) as a sequence of iterates. The method does not require that the nonlinearities be differentiable with respect to the dependent variable and its derivatives. The Adomian's Decomposition Method has been shown to solve effectively, easily, and accurately a large class of linear and nonlinear problems, generally two or three iterations lead to high accurate solutions. The basic motivation of the present study is to extend the application of Adomian's decomposition method to linear and nonlinear Goursat problems.

\section{Analysis of Adomian's Decomposition Method}

In order to explicate the solution procedure of the Adomian's Decomposition method (ADM), we consider the following nonlinear differential equation

$$
L_{x} L_{t} u(x, t)=f(x, t)+u^{n}(x, t),
$$

Where $L_{x}=\frac{\partial}{\partial x}, L_{t}=\frac{\partial}{\partial t}$, with the inverse operator $L_{x}{ }^{-1} L_{t}{ }^{-1}()=.\int_{0}^{x} \int_{0}^{t}() d x d$.

The solution of the problem is assumed as

$$
u(x, t)=\sum_{n=0}^{\infty} u_{n}(x, t),
$$

And the nonlinear function is considered as

$$
u^{n}(x, t)=\sum_{n=0}^{\infty} A_{n}(x, t),
$$

Where the $A_{n}(x, t)$, are called Adomian's polynomials which are determined by the following scheme

$$
\begin{gathered}
A_{0}=F\left(u_{0}\right), \\
A_{1}=u_{1} F^{\prime}\left(u_{0}\right), \\
A_{2}=u_{2} F^{\prime}\left(u_{0}\right)+\frac{u_{1}{ }^{2}}{2 !} F^{\prime \prime}\left(u_{0}\right), \\
A_{3}=u_{3} F^{\prime}\left(u_{0}\right)+u_{1} u_{2} F^{\prime \prime}\left(u_{0}\right)-\frac{u_{1}{ }^{3}}{3 !} F^{\prime \prime \prime}\left(u_{0}\right),
\end{gathered}
$$

and so on. The components of $u_{n}(x, t)$ are completely determined, by the recurrent relationship. 


$$
\begin{gathered}
u_{0}=f(x), \\
u_{1}=f(x)+L_{x} L_{t} A_{0}, \\
u_{n}=f(x)+\int_{0}^{x} \int_{0}^{t} A_{n-1} d x d t, n \geq 1 .
\end{gathered}
$$

Consequently, the exact solution may be obtained by using Eq. (2).

\section{Numerical Applications}

In this section, we apply Adomian's Decomposition Method (ADM) to solve linear and nonlinear Goursat problems. Numerical results are very encouraging.

Example 3.1. Consider the following linear homogeneous Goursat Problem

$$
u_{x t}=u,
$$

Subject to the conditions

$$
u(x, 0)=e^{x}, u(0, t)=e^{t}, u(0,0)=1 .
$$

According to procedure above

$$
\begin{gathered}
u(x, t)-u(0, t)-u(x, 0)+u(0,0)=\int_{0}^{x} \int_{0}^{t} u(x, t) d t d x, \\
u(x, t)=e^{x}+e^{t}-1+\int_{0}^{x} \int_{0}^{t} u(x, t) d t d x .
\end{gathered}
$$

Consequently, we have

$$
\begin{aligned}
& u_{0}(x, t)=e^{x}+e^{t}-1, \\
& u_{1}(x, t)=\int_{0}^{x} \int_{0}^{t} u_{0}(x, t) d t d x=\int_{0}^{x} \int_{0}^{t}\left[e^{x}+e^{t}-1\right] d t d x \\
& =x e^{t}+t e^{x}-x t-x-t, \\
& u_{2}(x, t)=\int_{0}^{x} \int_{0}^{t} u_{1}(x, t) d t d x \\
& =\int_{0}^{x} \int_{0}^{t}\left[x e^{t}+t e^{x}-x t-x-t\right] d t d x \\
& =\frac{x^{2}}{2} e^{t}+\frac{t^{2}}{2} e^{x}-\frac{x^{2}}{2} \frac{t^{2}}{2}-\frac{x^{2}}{2} t-\frac{t^{3}}{3 !}-\frac{x^{2}}{2}-\frac{t^{2}}{2}, \\
& u_{3}(x, t)=\int_{0}^{x} \int_{0}^{t} u_{2}(x, t) d t d x \\
& =\int_{0}^{x} \int_{0}^{t}\left[\frac{x^{2}}{2} e^{t}+\frac{t^{2}}{2} e^{x}-\frac{x^{2}}{2} \frac{t^{2}}{2}-\frac{x^{2}}{2} t\right. \\
& \left.-\frac{t^{3}}{3 !}-\frac{x^{2}}{2}-\frac{t^{2}}{2}\right] d x d t \\
& =\frac{x^{3}}{3 !} e^{t}+\frac{t^{3}}{3 !} e^{x}-\frac{x^{3}}{3 !} \frac{t^{3}}{3 !}-\frac{x^{3}}{3 !} \frac{t^{2}}{2 !}-\frac{t^{4}}{4 !}-\frac{x^{3}}{3 !} t-x \frac{t^{3}}{3 !}-\frac{x^{3}}{3 !}-\frac{t^{3}}{3 !},
\end{aligned}
$$

The series solution is

$$
\begin{aligned}
u(x, t)=e^{x}+ & e^{t}-1+x e^{t}+t e^{x}-x t-x-t+\frac{x^{2}}{2} e^{t} \\
& +\frac{t^{2}}{2} e^{x}-\frac{x^{2}}{2} \frac{t^{2}}{2}-\frac{x^{2}}{2} t-\frac{t^{3}}{3 !}-\frac{x^{2}}{2}-\frac{t^{2}}{2} \\
& +\frac{x^{3}}{3 !} e^{t}+\frac{t^{3}}{3 !} e^{x}-\frac{x^{3}}{3 !} \frac{t^{3}}{3 !}-\frac{x^{3}}{3 !} \frac{t^{2}}{2 !}-\frac{t^{4}}{4 !} \\
& -\frac{x^{3}}{3 !} t-x \frac{t^{3}}{3 !}-\frac{x^{3}}{3 !}-\frac{t^{3}}{3 !}+\cdots
\end{aligned}
$$

$$
\begin{gathered}
u(x, t)=e^{x}\left(1+t+\frac{t^{2}}{2 !}+\cdots\right)+e^{t}\left(1+x+\frac{x^{2}}{2 !}+\cdots\right) \\
-\left(1+(x+t)+\frac{(x+t)^{2}}{2 !}+\cdots\right)
\end{gathered}
$$

The closed form solution is

$$
u(x, t)=e^{(x+t)} .
$$

Example 3.2. Consider the following linear inhomogeneous Goursat Problem

$$
u_{x t}=u-t
$$

Subject to the conditions

$$
u(x, 0)=e^{x}, u(0, t)=t+e^{t}, u(0,0)=1 .
$$

According to the procedure defined above, we have

$$
\begin{aligned}
& u(x, t)= u(0, t)+u(x, 0)-u(0,0)-\frac{t^{2}}{2} \\
&+\int_{0}^{x} \int_{0}^{t} u(x, t) d t d x \\
&=t+e^{t}+e^{x}-1-\frac{t^{2}}{2}+\int_{0}^{x} \int_{0}^{t} u(x, t) d t d x .
\end{aligned}
$$

Consequently, we have

$$
\begin{gathered}
u_{0}(x, t)=t+e^{t}+e^{x}-1-\frac{t^{2}}{2} \\
u_{1}(x, t)=\int_{0}^{x} \int_{0}^{t} u_{0}(x, t) d t d x \\
=\int_{0}^{x} \int_{0}^{t}\left[t+e^{t}+e^{x}-1-\frac{t^{2}}{2}\right] d t d x \\
=x \frac{t^{2}}{2}+x e^{t}+t e^{x}-x t-x \frac{t^{3}}{3 !}-x-t, \\
u_{2}(x, t)=\int_{0}^{x} \int_{0}^{t}\left[x \frac{t^{2}}{2}+x e^{t}+t e^{x}-x t-x \frac{t^{3}}{3 !}-x\right. \\
-t] d t d x \\
=\frac{x^{2}}{2} \frac{t^{3}}{3 !}+\frac{x^{2}}{2} e^{t}+\frac{t^{2}}{2} e^{x}-\frac{x^{2}}{2} \frac{t^{2}}{2}-\frac{x^{2}}{2} \frac{t^{4}}{4 !}-\frac{x^{2}}{2} t-x \frac{t^{2}}{2}-\frac{x^{2}}{2}-\frac{t^{2}}{2}, \\
\text { and so on. }
\end{gathered}
$$

The series solution is 


$$
\begin{aligned}
u(x, t)=t+e^{t} & +e^{x}-1-\frac{t^{2}}{2}+x \frac{t^{2}}{2}+x e^{t}+t e^{x}-x t \\
& -x \frac{t^{3}}{3 !}-x-t+\frac{x^{2}}{2} \frac{t^{3}}{3 !}+\frac{x^{2}}{2} e^{t}+\frac{t^{2}}{2} e^{x} \\
& -\frac{x^{2}}{2} \frac{t^{2}}{2}-\frac{x^{2}}{2} \frac{t^{4}}{4 !}-\frac{x^{2}}{2} t-x \frac{t^{2}}{2}-\frac{x^{2}}{2}-\frac{t^{2}}{2} \\
& +\cdots \\
u(x, t) & =t+e^{t}\left(1+x+\frac{x^{2}}{2 !}+\cdots\right) \\
+ & e^{x}\left(1+t+\frac{t^{2}}{2 !}+\cdots\right)-(1+(x+t) \\
+ & \left.\frac{(x+t)^{2}}{2 !}+\cdots\right)
\end{aligned}
$$

The close form solution is

$$
u(x, t)=t+e^{(x+t)} .
$$

Example 3.3. Consider the following linear inhomogeneous Goursat Problem

$$
u_{x t}=u+4 x t-x^{2} t^{2}
$$

Subject to the conditions

$$
u(x, 0)=e^{x}, u(0, t)=e^{t}, u(0,0)=1 .
$$

According to the procedure defined above, we have

$$
\begin{gathered}
u(x, t)=u(0, t)+u(x, 0)-u(0,0)+4 \frac{x^{2}}{2} \frac{t^{2}}{2}-\frac{x^{3}}{3} \frac{t^{3}}{3} \\
+\int_{0}^{x} \int_{0}^{t} u(x, t) d t d x \\
=e^{x}+e^{t}-1+4 \frac{x^{2}}{2} \frac{t^{2}}{2}-\frac{x^{3}}{3} \frac{t^{3}}{3}+\int_{0}^{x} \int_{0}^{t} u(x, t) d t d x,
\end{gathered}
$$

Consequently, we have

$$
\begin{array}{r}
u_{0}(x, t)=e^{x}+e^{t}-1+4 \frac{x^{2}}{2} \frac{t^{2}}{2}-\frac{x^{3}}{3} \frac{t^{3}}{3} \\
u_{1}(x, t)=\int_{0}^{x} \int_{0}^{t} u_{0}(x, t) d t d x \\
=\int_{0}^{x} \int_{0}^{t}\left[e^{x}+e^{t}-1+4 \frac{x^{2}}{2} \frac{t^{2}}{2}\right. \\
\left.-\frac{x^{3}}{3} \frac{t^{3}}{3}\right] d t d x \\
=t e^{x}+x e^{t}-x t+\frac{x^{3} t^{3}}{9}-\frac{x^{4} t^{4}}{144}-x-t
\end{array}
$$

The series solution is

$$
\begin{gathered}
u(x, t)=e^{x}+e^{t}-1+4 \frac{x^{2}}{2} \frac{t^{2}}{2}-\frac{x^{3}}{3} \frac{t^{3}}{3}+t e^{x}+x e^{t}-x t \\
+\frac{x^{3} t^{3}}{9}-\frac{x^{4} t^{4}}{144}-x-t+\cdots \\
=x^{2} t^{2}+e^{x}\left[1+t+\frac{t^{2}}{2 !}+\cdots\right]+e^{t}\left[1+x+\frac{x^{2}}{2 !}+\cdots\right] \\
-\left[1+(x+t)+\frac{(x+t)^{2}}{2 !}+\cdots\right]
\end{gathered}
$$

The closed form solution is

$$
u(x, t)=x^{2} t^{2}+e^{(x+t)} .
$$

Example 3.4. Consider the following non-linear inhomogeneous Goursat Problem

$$
u_{x t}=-u^{3}+x^{3}+3 x^{2} t+3 x t^{2}+t^{3},
$$

Subject to the solution

$$
u(0, t)=t, u(x, 0)=x, u(0,0)=0 .
$$

According to the procedure defined above, we have

$$
\begin{gathered}
u(x, t)=x+t+\frac{x^{4} t}{4}+\frac{x^{3} t^{2}}{2}+\frac{x^{2} t^{3}}{2}+\frac{x t^{4}}{4} \\
-\int_{0}^{x} \int_{0}^{t} u^{3}(x, t) d t d x \\
=x+t+\frac{x^{4} t}{4}+\frac{x^{3} t^{2}}{2}+\frac{x^{2} t^{3}}{2}+\frac{x t^{4}}{4}-\int_{0}^{x} \int_{0}^{t} A_{n} d t d x
\end{gathered}
$$

Consequently, we have

$$
u_{0}(x, t)=x+t,
$$

$u_{1}(x, t)=\frac{x^{4} t}{4}+\frac{x^{3} t^{2}}{2}+\frac{x^{2} t^{3}}{2}+\frac{x t^{4}}{4}-\int_{0}^{x} \int_{0}^{t} A_{0} d t d x$

$$
=\frac{x^{4} t}{4}+\frac{x^{3} t^{2}}{2}+\frac{x^{2} t^{3}}{2}+\frac{x t^{4}}{4}-\int_{0}^{x} \int_{0}^{t}(x+t)^{3} d t d x
$$

$=\frac{x^{4} t}{4}+\frac{x^{3} t^{2}}{2}+\frac{x^{2} t^{3}}{2}+\frac{x t^{4}}{4}-\left(\frac{x^{4} t}{4}+\frac{x^{3} t^{2}}{2}+\frac{x^{2} t^{3}}{2}+\frac{x t^{4}}{4}\right)=0$,

and so on.

The solution is

$$
u(x, t)=\sum_{n=0}^{\infty} u_{n}=x+t .
$$

Example 3.5. Consider the following non-linear Inhomogeneous Goursat Problem

$$
u_{x t}=-u^{2}+e^{2 x}+e^{2 t}+2 e^{x+t}
$$

With the condition

$$
u(x, 0)=1+e^{x}, u(0, t)=1+e^{t}, u(0,0)=2 .
$$

According to the procedure defined above, we have

$$
\begin{gathered}
u(x, t)=e^{x}+e^{t}+\frac{x t e^{2 t}}{2}+\frac{t e^{2 x}}{2}-\frac{x e^{2 t}}{4}+\frac{x}{4}+2 e^{(x+t)} \\
-2 e^{x}-2 e^{t}-\frac{t}{2}+2-\int_{0}^{x} \int_{0}^{t} u^{2} d t d x \\
=e^{x}+e^{t}+\frac{x e^{2 t}}{2}+\frac{t e^{2 x}}{2}-\frac{x}{2}+2 e^{(x+t)}-2 e^{x}-2 e^{t}+2- \\
\int_{0}^{x} \int_{0}^{t} A_{n} d t d x .
\end{gathered}
$$

Consequently, we have

$$
\begin{gathered}
u_{0}=e^{x}+e^{t}, \\
u_{1}(x, t)=\frac{x e^{2 t}}{2}+\frac{t e^{2 x}}{2}-\frac{x}{2}+2 e^{(x+t)}-2 e^{x}-2 e^{t}+2 \\
-\int_{0}^{x} \int_{0}^{t} A_{0} d t d x
\end{gathered}
$$




$$
\begin{gathered}
=\frac{x e^{2 t}}{2}+\frac{t e^{2 x}}{2}-\frac{x}{2}+2 e^{(x+t)}-2 e^{x}-2 e^{t}+2-\int_{0}^{x} \int_{0}^{t}\left(e^{x}+\right. \\
\left.e^{t}\right)^{2} d t d x=0,
\end{gathered}
$$

and so on.

The solution is

$$
u(x, t)=\sum_{n=0}^{\infty} u_{n}=e^{x}+e^{t} .
$$

\section{Conclusions}

We have successfully applied the Adomian's decomposition method for linear and nonlinear solving Goursat problems. This method can also be extended to other nonlinear partial differential equations. The method is applied in a direct way without using linearization, transformation, discretization or restrictive assumptions. It may be concluded that the proposed method is very powerful and efficient in finding the analytical solutions for a wide class of boundary value problems.

\section{REFERENCES}

[1] J.T. Day, Runge-Kutta method for the numerical solution of the Goursat problem in hyperbolic partial differential equations. Comput. T. 9: (1966), 81-83.

[2] D. J. Evans and B.B. Sangui, Numericalsolution of the Goursat problem by a nonlineartrapezoidal formula. Appl. Math. Lett., 1: (1988), 221-223.

[3] A. M.Wazwaz, On the numerical solution oftheGoursat problem, Appl. Math. Comput., 59: (1993), 89-95.

[4] A. M.Wazwaz, The decomposition methodfor the approximate solution to the Goursatproblem. Appl. Math. Comput., 69: (1995), 299-311.

[5] A. M.Wazwaz, The variationaliterationmethod for the exact solution of Laplace equation.Phys. Lett., A 363: (2007), 260-262.

[6] A. M.Wazwaz, The variationaliterationmethod for the reliable treatment of linear andnonlinearGoursat problems. Appl. Math. Comput.,193: (2007), 455-462.

[7] Adomian, A review of the decomposition method in Applied Mathematics, J. Math. Anal., 135 (1988), 501-544.

[8] Adomian, Solving Frontier Problems of Physics: The Decomposition Method, Kluwer Academic Publishers, Boston, (1994).

[9] A. M. Wazwaz, A First Course in Integral Equations, World Scientific, Singapore, 1997.

[10] A. M. Wazwaz, A new algorithm for calculating Adomian polynomials for nonlinear operators, 111 (2000), 53-69.

[11] J. Ahmad, F. Hussain and M. Naeem, Laplace Decomposition Method for Solving Singular Initial Value Problems, Aditi Journal of Mathematical Physics, 5(1)1-15, 2014.

[12] G. Adomian, The decomposition method for nonlinear dynamic systems, J. Math. Anal. Appl., 120: (1986), 270-283.

[13] B. Datta, A new approach to the wave equationan application of the decomposition method, J. Math. Anal. Appl., 1 (142), (1987), 6-12

[14] J. Ahmad and S. T.Mohyud-Din, Solving Fractional Vibrational Problem Using Restarted Fractional Adomian's Decomposition Method, Life Sci J; 10(4): (2013), 210-216.

[15] G. Adomian, Solution of coupled nonlinear partial differential equations by decomposition, Comp. Math. Appl., 6 (31): (1995), 117-120. 